\title{
Toward dynamic ptychography using a spatiotemporal overlap constraint
}

\author{
G. Hinsley ${ }^{1, *}$, G. van Riessen ${ }^{1}$, C. M. Kewish ${ }^{1,2}$ \\ ${ }^{1}$ Department of Chemistry and Physics, La Trobe Institute for Molecular Science, La Trobe University, Melbourne, Victoria 3086, \\ Australia, \\ ${ }^{2}$ ANSTO Australian Synchrotron, Clayton VIC 3168, Australia \\ *G.Hinsley@ltu.edu.au
}

Coherent imaging methods such as ptychography have a growing interest for multidimensional imaging applications due to the high spatial resolution at which quantitative information is obtained. In the application of X-ray imaging to the study of dynamic processes [1], the achievable temporal resolution is limited by detector performance. However, for ptychography this is also determined by the degree of redundancy in the diffraction data that is needed to reliably reconstruct real-space images. The success of ptychography lies in the incorporation of redundancy in the spatial dimensions from transverse translation diversity of the object, which allows for relaxation of constraints on the illumination and other experimental factors. Other schemes such as longitudinal translation (phase) diversity [2], and probe diversity [3], have been reported.

Using the time dimension redundancy for the introduction of a constraint has recently been proposed in coherent diffractive imaging (CDI) [4,5], exploiting "overlap" between successive images to achieve similar advantages to ptychography. However, these methods impose limits on the object or illumination and require a priori knowledge of the location of time-independent regions of the object, making them incompatible with scanning methods such as ptychography. We have developed an algorithm that removes these limitations and introduces redundancy without guidance and without requiring reference object regions by exploiting intentional or incidental temporal diversity in the diffraction data [6]. Our approach automatically defines a spatiotemporal constraint through automatically segmenting time-dependent and time-independent regions within the image field, dependent on the detected sample dynamics, and is able to suppress ambiguity and artefacts in the reconstructions.

Spatiotemporal redundancy in time-series coherent diffraction data provides a viable path toward studying nanoscale dynamics by Xray imaging. We demonstrate this potential through CDI simulations of different dynamic phenomena under realistic conditions modelled on the XFM beamline at the Australian Synchrotron, the motion of nanoparticles, as well as the oscillatory behaviour of a 2dimensional chemical reaction. An extension to dynamic ptychography that allows high quality reconstructions to be achieved with a relaxed spatial overlap constraint and faster scanning is then examined through simulation. Preliminary experimental investigation of crack propagation within thin metallic films, obtained using the EIGER2 detector at the XFM beamline, will be presented.

This work was performed in part at the Melbourne Centre for Nanofabrication (MCN) in the Victorian Node of the Australian National Fabrication Facility (ANFF). This research was undertaken on the XFM beamline at the Australian Synchrotron, part of ANSTO.

[1] J. Lim et al., "Origin and hysteresis of lithium compositional spatiodynamics within battery primary particles,” Science, 353, 566 (2016).

[2] C. T. Putkunz, et al., "Phase-Diverse Coherent Diffractive Imaging: High Sensitivity with Low Dose,” Phys. Rev. Lett. 106, 013903 (2011).

[3] I. Peterson, et al., "Probe-diverse ptychography", Ultramicroscopy 171, 77 (2016).

[4] Y. H. Lo, et al., "In situ coherent diffractive imaging," Nat. Commun. 9, 1826 (2018)

[5] X. Tao, et al., "Spatially correlated coherent diffractive imaging method," Appl. Opt. 57, 6527 (2018).

[6] G. N. Hinsley, et al., "Dynamic Coherent Diffractive Imaging Using Unsupervised Identification Of Spatiotemporal Constraints," Opt. Express 28, $36862(2020)$.

Keywords: Dynamic; spatiotemporal constraint; coherent diffractive imaging; nanoparticle, ptychography; 